\title{
Activismo por los derechos digitales en América Latina Pensar globalmente, actuar localmente ${ }^{1}$
}

\author{
María Soledad Segura ${ }^{2}$
}

Recibido: 1 de agosto, 2019

Aceptado: 27 de octubre, 2019

\section{RESUMEN}

Analizaré cómo actores progresistas de la sociedad civil presionan para la formulación de políticas públicas basadas en los derechos digitales, que ponen límites a los abusos del Estado y del mercado en la comunicación pública, y que protegen y garantizan los derechos de los ciudadanos a la comunicación y la cultura. El activismo por los derechos digitales en América Latina está representado por movimientos sociales que promueven políticas públicas basadas en los derechos al acceso, producción y gestión digital y de datos, frente a los abusos de corporaciones, gobiernos y organizaciones criminales. Se debate si los desafíos generados por una tecnología global serían los mismos a los de los países centrales, o si cabe distinguir particularidades regionales; si los usos de estas relativamente nuevas tecnologías, implican también la aparición de problemas nuevos, o si se trata de la reiteración de antiguos problemas; y si, por ende, estamos ante antiguos o nuevos marcos interpretativos, demandas, oponentes y aliados, estrategias, y tipos de organizaciones para enfrentarlos en Latinoamérica.

Palabras clave | activismo digital, derechos digitales, políticas de digitalización, demandas, impacto.

\footnotetext{
${ }^{1}$ Se agradece el asesoramiento de Nicolás Wolovick para la elaboración de este artículo.

${ }^{2}$ Doctora en Ciencias Sociales, profesora de la Universidad Nacional de Córdoba e investigadora del Consejo Nacional de Investigaciones Científicas y Técnicas de Argentina, y autora de: De la resistencia a la incidencia. Sociedad civil y derecho a la comunicación en la Argentina (Ediciones UNGS, 2018). maria.soledad.segura@unc.edu.ar
} 


\section{ABSTRACT}

\section{Activism for digital rights in Latin America. Think globally, act locally}

I will analyze how progressive civil society actors push for the formulation of public policies based on digital rights, which put limits on state and market abuses in public communication, and that protect and guarantee citizens' rights to communication and culture. The activism for digital rights in Latin America is represented by social movements that promote rights-based public policies regarding digital and data access, production and management, against abuses by corporations, governments and criminal organizations. It is debated whether the challenges generated by a global technology would be the same as those of the central countries, or if regional particularities can be distinguished; if the uses of these relatively new technologies, also imply the appearance of new problems, or if it is the repetition of old problems; and if, therefore, we are facing old or new interpretive frameworks, demands, opponents and allies, strategies, and types of organizations to deal with them in Latin America.

Keywords | Digital activism, digital rigths, digital policies, demands, impact.

\section{INTRODUCCIÓN}

El análisis de las experiencias recientes de activismo digital en América Latina muestran que existen dos tipos diferenciados por objetivos y esferas de acción: el activismo digital social que utiliza diversas tácticas para el cambio social, incluida la recolección, producción y manejo de información, y el razonamiento basado en big data; y el activismo por los derechos digitales que está representado por movimientos sociales que promueven la vigencia y ampliación de derechos con respecto a la producción, protección y gestión de datos, y tienen como objetivo regular el extractivismo de datos por parte de corporaciones y estados. Este último hace referencia a actores progresistas de la sociedad civil que presionan para la formulación de políticas públicas basadas en los derechos digitales, que ponen límites a los abusos del estado y del mercado en la comunicación pública, y que protegen y garantizan los derechos de los ciudadanos a la comunicación y la cultura. El activismo por los derechos digitales en la región está representado por movimientos sociales que promueven políticas públicas basadas en los derechos con respecto al acceso, 
María Soledad Segura

producción y gestión digital y de datos, frente a los abusos de corporaciones, gobiernos y organizaciones criminales (Segura y Waisbord, 2019).

Si bien en los últimos años en América Latina se han realizado múltiples trabajos sobre políticas de telecomunicaciones (Regulatel, 2016; Mastrini y Aguerre, 2009; Mariscal y Rivera, 2007; Rivera, 2004; entre otros/as), sobre la brecha digital en la región (CEPAL, 2016; SITEAL, 2012), y algunos sobre activismo digital social (Gutiérrez, 2018; Chenou y Cepeda-Másmela, 2019; Millaleo y Velasco, 2013), todavía hay escasos antecedentes de investigaciones sobre el activismo por los derechos digitales y de datos en esta parte del mundo.

Este artículo se centra en el análisis de las dimensiones del activismo por los derechos digitales en América Latina. No se pretende realizar un relevamiento exhaustivo de las organizaciones que trabajan en este sentido, sino identificar las principales tendencias y, en base a ellas, discutir si los desafíos generados por una tecnología global son los mismos en todas las regiones del planeta, o si cabe distinguir particularidades locales o regionales diferentes a las de los países centrales (Segura y Waisbord, 2019). También se propone analizar si los usos de estas relativamente nuevas tecnologías, implican también la aparición de problemas nuevos, o si se trata de la reiteración de antiguos problemas; y si, por ende, estamos ante antiguos o nuevos marcos interpretativos, demandas, oponentes y aliados, estrategias, y tipos de organizaciones para enfrentarlos en nuestra región.

Se argumenta lo siguiente: Frente a los problemas en el uso de las tecnologías de la digitalización y la conectividad en el capitalismo digital, las organizaciones de activistas por los derechos digitales en América Latina son actores progresistas de la sociedad civil que tienen por propósito garantizar los derechos humanos a la comunicación (acceso a la información y libertad de expresión), la cultura y la privacidad; que entienden que, para ello, es necesario reducir los abusos de poder de gobiernos y empresas en la comunicación pública digital; y que, con ese fin, desarrollan dos tipos de estrategias: por un lado, presionan para la formulación de políticas públicas basadas en derechos $y$, por otro, desarrollan prácticas autogestivas para ejercer estos derechos de hecho, estén o no reconocidos legalmente.

La hipótesis planteada es que el activismo digital y de datos recorre los caminos ya trazados por el activismo comunicacional y cultural en la era analógica sobre modalidades de intervención en lo público, y de relación 
Vol. XXXIII | N 2 julio - diciembre 2019| ISSN 0719-0883 | pp. 198-228

conflictiva con el poder estatal y el mercantil. Por lo tanto, la disputa por lo digital no modifica -en lo esencial- las dinámicas y los poderes estructurales de la comunicación pública.

El texto se estructura del siguiente modo: Inicialmente, explicitaré cuál es el enfoque teórico a partir del cual se produce el análisis. Luego, para responder las preguntas propuestas, comprobar la hipótesis y desarrollar el argumento, analizaré, primero, cuáles son las demandas y el marco interpretativo de las organizaciones de activistas por los derechos digitales; en segundo lugar, los problemas que enfrentan, sus oponentes y potenciales aliados; en tercer término, qué estrategias desarrollan; en cuarto lugar, cuáles son los tipos de organizaciones existentes y sus características generales; $y$, finalmente, el impacto que consiguieron.

\section{ENFOQUE TEÓRICO}

Se abordan las preguntas planteadas, considerando que la formulación de políticas públicas es un proceso conflictivo que involucra a varios actores sociales con diferentes intereses, estrategias, recursos y poderes (Freedman, 2008). El Estado se concibe, entonces, como una entidad diferenciada, compleja y contradictoria, como terreno de disputas para trazar esas políticas (Oszlak y O'Donnell, 1984).

También es necesario señalar que la sociedad civil que procura incidir en la formulación de políticas públicas al tiempo que desarrolla sus propias políticas, no es un todo unificado, sino que es compleja, conflictiva y heterogénea. No se adscribe a la visión de una esfera homogénea ni pura, sede de la libertad frente a la opresión estatal, y del altruismo frente a la dominación del mercado. Por el contrario, se entiende que mantiene una relación de autonomía relativa con el Estado y el mercado (Sorj, 2010).

En particular, el activismo por los derechos digitales es una forma de acción colectiva que promueve el acceso y la gestión más igualitarios de la tecnología y de los datos, se involucra con nuevas formas de producción de información y conocimiento, y cuestiona los conceptos dominantes sobre la digitalización de información y la conectividad (Milan y van der Velden, 2016). 
María Soledad Segura

Se analiza las dimensiones del activismo por los derechos digitales según las teorías sociológicas de los movimientos sociales que se enfocaron en tres aspectos centrales: el contexto de surgimiento de los movimientos; su particular forma de organizarse, actuar y comunicar; y el impacto que generan. Dado que la hipótesis propuesta es que el activismo digital recorre caminos ya conocidos por el activismo comunicacional y cultural en la era analógica, también se quiere mostrar en este artículo que el enfoque teórico de movimientos sociales sigue siendo operativo para identificar las principales dimensiones analíticas de este fenómeno: los problemas que enfrenta, las demandas que presenta, el marco interpretativo desde el que formula sus demandas, sus estrategias, sus organizaciones, el contexto en que surge, las oportunidades de acción e incidencia que se le presentan, y sus niveles de incidencia (Tarrow, 1977).

Finalmente, es relevante destacar que no se concibe a la democracia y los derechos como un fin predefinido que se alcanza o no se alcanza totalmente de una vez y para siempre. Por el contrario, se plantea la existencia de un "proceso de democratización" y de un "proceso de ampliación de derechos", porque esto permite identificar los matices en los tipos de acciones y sus alcances, los avances y los retrocesos, los diversos niveles en que cada derecho se amplía o se restringe, las contradicciones y paradojas. Es más, en el mismo proceso se definen y redefinen los sentidos otorgados a la democracia y a los derechos que están en disputa (Fraser, 2006).

\section{MARCO INTERPRETATIVO}

En esta sección se responden las siguientes interrogantes: ¿Cuáles son las demandas que presenta el activismo digital y de datos en América Latina, y desde qué marco interpretativo las formula? Estas demandas y marcos interpretativos, ¿son nuevos y específicos de la era digital o son antiguos y comunes a la era analógica? Y ¿son propios de Latinoamérica o retoman tradiciones de los países centrales?

Las organizaciones de la sociedad civil latinoamericana que trabajan por el respeto y ampliación de los derechos afectados por los usos de las tecnologías de digitalización y conectividad, trabajan en tres áreas temáticas: (1) el derecho a la comunicación en su doble dimensión: a la libertad de expresión y no censura ni inducción a la auto-censura, y a la producción, acceso y verificación de la información pública, verificación de identidades de los participantes en la 
Vol. XXXIII | N² 2 julio - diciembre 2019 | ISSN 0719-0883 | pp. 198-228

deliberación pública y no discriminación (neutralidad de la red); (2) los derechos culturales de acceso y conservación del patrimonio digital; y (3) el derecho a la privacidad, al olvido y a no ser controlado ni vigilado.

Estos derechos retoman la tradición del derecho humano a la comunicación y la cultura basado en los principios de acceso, pluralidad, diversidad, participación, y equidad, referidos tanto al acceso y uso de las tecnologías de la digitalización y la conectividad mismas, como a la producción, acceso y gestión de datos privados y públicos. El derecho a la comunicación es un derecho que incluye y supera los de libertad de expresión (no censura ni inducción a la autocensura) y acceso a la información pública. Al aplicarse a nuevas tecnologías, enfrentan nuevos desafíos que obligan a redefiniciones, precisiones y ampliaciones de estos derechos. Los activistas por los derechos digitales amplían y especifican las implicaciones de estos principios en respuesta a los desafíos que plantean las nuevas posibilidades tecnológicas. Una de esas novedades es el principio de neutralidad de la red que establece que todos los contenidos que circulan por Internet deben recibir el mismo trato y no ser discriminados por su origen, uso o aplicación (Wu, 2003).

En materia de derechos culturales, basados en los mismos principios que el derecho a la comunicación, se promueven especialmente el libre acceso -con un reconocimiento de los derechos de autor compatible con el libre acceso- y la producción colaborativa que la tecnología permite. Por otra parte, hay poblaciones que reivindican su derecho a la no digitalización de sus bienes culturales para evitar que les sean robados con fines comerciales.

Retoman también los derechos civiles a la privacidad frente al control de estados y el uso de datos privados con fines comerciales, e introducen novedades como el derecho al olvido, esto es: a que la información personal puesta en disponibilidad pública de manera voluntaria pueda también ser retirada cuando la persona así lo desee.

Por ende, la sociedad civil procura reducir las prácticas abusivas de gobiernos y corporaciones, los otros dos históricos sectores con mayor poder, en la comunicación y cultura públicas, así como en el manejo de datos personales. Aunque se debate el rol del Estado, muchas organizaciones de la región -en línea con las demandas por el derecho a la comunicación en materia audiovisual, por ejemplo- consideran que el Estado debe intervenir para garantizar los derechos 
María Soledad Segura

civiles, culturales y a la comunicación.

De este modo, el activismo digital en la región abreva en los marcos legales progresistas y los principios políticos basados en las tradiciones filosóficas, éticas y políticas occidentales originadas en Europa y Estados Unidos, no propios de la región, y que están plasmados en la legislación internacional establecida por organismos multilaterales a nivel global; y los actualiza de acuerdo a los nuevos desafíos que los usos de las posibilidades tecnológicas presentan. Además, algunas de las innovadoras precisiones realizadas tienen origen en organizaciones de la región que las formulan teniendo en cuenta los contextos e historia locales.

\section{PROBLEMAS ${ }^{3}$}

Con ese marco interpretativo, ¿cuáles son los tipos de problemas que identifica y enfrenta el activismo digital y de datos en la región? Estos problemas, ¿son específicos de América Latina o son globales como la extensión de las tecnologías de digitalización y conectividad? ¿Son específicos del llamado capitalismo digital o actualizan antiguos problemas de la comunicación y la cultura públicas?

El activismo por los derechos digitales enfrenta antiguos problemas relativos a violaciones a los derechos a la comunicación, la cultura y la privacidad que, ante las nuevas posibilidades tecnológicas de la digitalización y la conectividad, adquieren otras dimensiones y particularidades. Además, en la región -del mismo modo que en gran parte de los países periféricos- la demanda de acceso a la tecnología para saldar la brecha digital sigue teniendo vigencia.

\section{Problemas de acceso a la tecnología digital y la conectividad}

En América Latina, 50\% de la población no tiene acceso a Internet o tiene mala calidad de conexión. Hay mucha desigualdad entre zonas rurales y urbanas, varones y mujeres, jóvenes y viejos, etc. (CEPAL, 2017):

“La persistencia de las desigualdades digitales en América Latina (...) nos recuerda que el capitalismo no está igualmente interesado en la

\footnotetext{
${ }^{3}$ No se pretende aquí caracterizar exhaustivamente al capitalismo digital y de vigilancia en América Latina, sino que sólo se mencionan los problemas de este sistema que los/as activistas digitales identifican como tales a partir de su marco interpretativo y que enfrentan con diversas tácticas y estrategias.
} 
Vol. XXXIII | N 2 julio - diciembre 2019| ISSN 0719-0883 | pp. 198-228

codificación de datos de todos los grupos sociales, ya sea con fines comerciales o políticos. (...) La exclusión digital confirma el interés selectivo del capitalismo en crear mercados y explotar el trabajo. (...) La fuerte promoción del extractivismo de datos, impulsado por el expansionismo corporativo o las políticas estatales, habría mejorado el acceso digital. Sin embargo, al igual que las formas anteriores del capitalismo, el capitalismo digital se dirige selectivamente a unos públicos mientras ignora completamente a los demás" (Segura y Waisbord, 2019: 5).

Esta enorme brecha en el acceso digital de calidad es otra forma de exclusión social en una región con altísimos niveles de desigualdad.

\section{Problemas referidos al derecho a la comunicación}

Es posible diferenciar tres grupos de problemas referidos al secretismo de la información pública por parte de estados y empresas, la censura privada de contenidos por parte de las plataformas, y la desinformación por difusión de noticias falsas, noticias engañosas, hechos alternativos, hostigamientos y discursos de odio.

En primer lugar, la apropiación de datos de interés público con fines políticos o comerciales que hacen los Estados y las corporaciones es una problemática antigua. Las tecnologías de digitalización y conectividad se supone que permitirían facilitar el acceso a la información pública porque posibilitan poner datos en línea y que resulten fácilmente utilizables. No obstante, es altamente difícil encontrar entidades públicas o privadas que produzcan y/o gestionen información pública que tengan políticas proactivas de acceso a la información y pongan los datos disponibles en línea y fácilmente accesibles y comprensibles.

En segundo término, los altísimos niveles de concentración de la propiedad de los distribuidores de contenidos por Internet (Facebook, Twitter, Instagram), sitúa a los intermediarios de internet en una posición de poder para definir qué discursos circulan y cuáles serán censurados. Por eso, adquieren el rol de cuello de botella, gatekeepers privados, o policía privada de contenidos. Justifican este rol censurador y discriminador de ciertos contenidos y plataformas, con la necesidad de enfrentar lo que denominan "noticias falsas". Sin embargo, esto sólo restringe la libertad de expresión y empodera aún más a los concentrados medios tradicionales. 
María Soledad Segura

En tercer lugar, la desinformación por difusión de discursos del odio, hostigamiento, noticias falsas ( $\sin$ base fáctica) o engañosas (descontextualizadas), hechos alternativos (que cuestionan comprobaciones científicas, como los terraplanistas o los anti-vacunas; o quienes desconocen hechos históricos como el holocausto judío, el genocidio armenio o los 30.000 desaparecidos en Argentina). Sin embargo, las campañas de desinformación no son novedad, si no que "han sido una estrategia de los monopolios mediáticos tradicionales para amenazar y desmantelar las democracias durante años" (Coalizão Direitos na Rede, 2017) y se vinculan con "viejos y nuevos desequilibrios de poder relacionados con la concentración de la propiedad de los medios, monopolios en redes sociales y los intereses políticos de los Estados nacionales para controlar y manipular el discurso -dentro y fuera de sus fronteras" (Coalizão Direitos na Rede, 2017). Lo que sí es nuevo, son las posibilidades de difusión masiva o hiper-dirigida, inmediata y en red: lo que antes era acordado entre los gobernantes y los dueños de los grandes medios de masas, ahora se postea y repostea desde múltiples puntos.

El hostigamiento en la deliberación pública ha estado destinado a "embarrar la cancha”, orientar el encuadre con el que debe entenderse una cuestión pública, promover la desinformación, e inducir a la auto-censura. Muchas de estas acciones o ciberataques son ejecutadas por las llamadas cibertropas, las cuales constituyen "un problema complejo, no centralizado y del que participan una diversidad de actores entre los que se destacan trolls, influenciadores con cuentas reales e incluso a veces verificadas, bots y cuentas fakes. La organización celular y no vertical es al mismo tiempo una característica que impide adjudicar a una única organización o a un único call center la acción de estas cibertropas" (Amnesty International-Argentina, 2018: 8). Las campañas de agresión en Internet desarrolladas por cibertropas propician "la inhibición y la autocensura no sólo directa, es decir, de las personas violentadas, sino también el disciplinamiento del entorno por efecto del escarmiento, que se produce en personas afines o cercanas a las posiciones de las agredidas" (Amnesty International-Argentina, 2018: 8). 
Vol. XXXIII | N² 2 julio - diciembre 2019| ISSN 0719-0883 | pp. 198-228

"En este sentido, opera en los ciberataques una economía moral que apunta a disuadir directa e indirectamente de emitir o compartir discursos considerados inconvenientes, al punto de que su circulación suscitará acoso. La disciplina perseguida por las cibertropas es el silencio o el cambio del tema de conversación." (Amnesty International-Argentina, 2018: 8)

Está comprobado que quienes más agresiones reciben en las redes sociales son los pertenecientes a grupos más débiles. En Twitter, por ejemplo, las más agredidas son las mujeres $\mathrm{y}$, entre las mujeres, las africanas y asiáticas, constituyen uno de los grupos especialmente hostigados (Amnesty International-United Kingdom, 2017)

Dentro de este grupo de problemas, se incluye también la propagación de los llamados discursos del odio: las campañas de desinformación de grupos extremistas -como el Estado Islámico-, que generan "una narrativa maliciosa contra las estructuras o las instituciones de un Estado, con el objetivo de polarizar la opinión pública y dañar la cohesión social de un país, que es la base de una democracia liberal" (Lesaca, 2019: 1). Funcionan mediante redes o medios de comunicación no convencionales de reciente creación. Después, esas narrativas se distribuyen a través de cuentas de redes sociales anónimas y automatizadas. Las plataformas más utilizadas dependen de los países, pero Twitter, Facebook y YouTube son las más usadas y llegan a la mayor cantidad de audiencia. (Lesaca, 2019)

\section{Problemas referidos a derechos culturales}

Los activistas por los derechos digitales enfrentan dos grupos de problemas: por un lado, la privatización y comercialización de bienes y servicios culturales; y la no conservación del patrimonio cultural digital, incluido el software. El primero es un problema de vieja data, que cobra mayor relevancia por las esperanzas democratizadoras que activaron las posibilidades de digitalización y conectividad que permiten que los bienes y servicios culturales sean accesibles para quienes no lo eran en la era analógica por razones de distancia geográfica, por ejemplo. El segundo es también un problema de la época analógica referido a la conservación de archivos, pero se agrava en la era digital por la velocidad de innovación tecnológica que impide que archivos guardados con una versión tecnológica anterior, puedan ser leídos o reproducidos. 
María Soledad Segura

\section{Problemas referidos a los derechos civiles}

Entre estos, se identifican las violaciones del derecho a la privacidad y la vigilancia y control.

La violación de la privacidad se produce por diversos mecanismos. En primer lugar, por la recolección masiva, continua y sin permiso de datos personales (gustos, intereses, comportamientos, etc.), tanto por corporaciones privadas como las de redes sociales, medios digitales, comercio en línea, "nubes" o editores en línea; como por instituciones estatales. En segundo lugar, se registra una escasa protección de datos personales en bases de datos en línea de instituciones públicas y privadas. Por otra parte, se preserva la disponibilidad pública de información personal más allá del deseo de las personas, esto es: la información personal que, inicialmente, pudo ser compartida por voluntad propia de sus titulares, luego, cuando éstos ya no quieren que esté disponible públicamente, es difícil darle de baja.

Las tres condiciones anteriores hacen posible que se produzca un uso indebido y sin autorización de esa información personal en estrategias de publicidad hiper-dirigida con fines comerciales y políticos. Los casos comerciales son continuos y conocidos en las redes sociales, medios digitales y sitios de e-commerce. Al respecto, el caso político más resonante en América Latina ha sido el de la campaña electoral del ahora presidente de Brasil, Jair Bolsonaro.

Por otra parte, y ya vinculado a los derechos políticos, es posible la manipulación de resultados electorales (como reconoce el empleado de una empresa que fabrica máquinas de voto electrónico en el documental Hacking Democracy de HBO).

La introducción de sistemas de voto electrónico también permite la seria restricción de las posibilidades de fiscalización de la votación y la potencial violación del secreto del voto (como sugirió el presidente venezolano Nicolás Maduro el 17 de mayo de 2013 al referirse al resultado de las elecciones del 14 de abril: "900.000 compatriotas... 900.000, ya los tenemos, con cédula de identidad y todo... Y eso significó que la brecha fuera más corta. La brecha pudo haber sido buena"). 
Vol. XXXIII | N² 2 julio - diciembre 2019 | ISSN 0719-0883 | pp. 198-228

Por otra parte, la vigilancia digital tiene dos dimensiones: la que afecta los derechos civiles de los/as ciudadanos/as y la que afecta la soberanía y seguridad nacional.

Por un lado, el uso indebido de esa información para control y vigilancia estatal alcanza su paroxismo con el control biométrico de migraciones en Argentina y otros países del mundo, y que también se ha impuesto en muchos lugares de trabajo, estatales y privados. Esto comienza a ser expresamente prohibido en algunos lugares del mundo como la ciudad estadounidense de San Francisco, cuya legislatura prohibió el uso de tecnologías de reconocimiento facial el 15 de mayo de este año 2019, debido a los errores que comete y por violar los derechos civiles de las personas.

En Latinoamérica, hay países que están incorporando tecnología de control digital y de datos a gran escala. En México, en 2017, se conoció que el gobierno de Enrique Peña Nieto vigilaba, con tecnología de espionaje digital comprada a Israel, a quienes reclamaban por la matanza de Ayotzinapa. Además, fue denunciado por espiar ilegalmente a veinte de sus críticos más abiertos. En 2018, dos periodistas de Rio Doce, un canal de noticias independiente que cubre cárteles de la droga, recibieron mensajes de texto mezclados con malware Pegasus realizado por la firma israelí de spyware NSO Group, y después, su colega, el galardonado periodista mexicano y cofundador de Rio Doce, Javier Valdez, fue asesinado (Electronic Frontier Foundation, 2018). El mismo año, en Guatemala, el Nuevo Diario reveló una operación de espionaje vasta e ilegal realizada por el gobierno del Partido Patriota (2011-2015) contra activistas, empresarios, políticos, periodistas, diplomáticos y líderes sociales de ese país que lucharon contra la corrupción gubernamental (Electronic Frontier Foundation, 2018). En Argentina, el gobierno de Mauricio Macri también le compró a Israel una herramienta de vigilancia de redes sociales supuestamente para la prevención del terrorismo.

Por otra parte, la cuestión del control y vigilancia también se da a nivel internacional entre gobiernos. En 2013, las filtraciones del ex técnico de la CIA, Edward Snowden, permitió comprobar que el gobierno de Estados Unidos espiaba a millones de personas en el mundo, incluidos/as presidentes/as y primeros/as ministros/as, como la brasileña Dilma Rousseff y el mexicano Enrique Peña Nieto. 
María Soledad Segura

En síntesis, la mayoría de los problemas (secretismo, inducción a la autocensura, discriminación, control, violación de la privacidad, desigualdad) que identifica y enfrenta el activismo por los derechos digitales en América Latina son antiguos: su existencia es anterior a la introducción del uso masivo de estas tecnologías de la digitalización y la conectividad. Sin embargo, ahora adquieren nuevas dimensiones que es preciso atender para buscar soluciones específicas. Además, si bien se trata de problemas globales, en América Latina adquieren particularidades locales que requieren estudio preciso para poder desarrollar políticas adecuadas a la región dada su historia y coyuntura.

\section{OPONENTES Y ALIADOS}

Considerando los problemas identificados habida cuenta del marco interpretativo de derechos humanos, cabe preguntarse ¿̇uáles son los aliados a los que recurren y los oponentes que enfrentan los/as activistas por los derechos digitales en la región?

El activismo por los derechos digitales en América Latina, tal como ya se demostró, enfrenta problemas vinculados a los abusos de poder del mercado, de los gobiernos y también de organizaciones criminales en materia de comunicación y cultura públicas.

Persisten los crónicos problemas de comunicación pública que caracterizaron a la era analógica: altísimos niveles de concentración de la propiedad privada de medios y empresas de telecomunicaciones, centralización de la producción de contenidos de prensa y audiovisual en los principales centros urbanos, relaciones de mutua conveniencia entre medios y gobiernos, prácticas secretistas en el manejo de la información pública, faltas de protección a los/as periodistas, etc. (Segura y Waisbord, 2016). Como se mostró antes, muchos de estos problemas continúan y cobran nueva dimensión con las tecnologías de la digitalización y la conectividad.

Tal es el caso de la concentración del mercado latinoamericano de medios y telecomunicaciones que adquiere niveles altísimos. Las estrategias de las empresas de telecomunicaciones robustecieron la tendencia a una estructuración oligopólica de las actividades infocomunicacionales en América Latina. Esto aceleró la tendencia a la concentración "ya que la convergencia 
Vol. XXXIII | N 2 julio - diciembre 2019| ISSN 0719-0883 | pp. 198-228

tecnológica entre medios, telecomunicaciones e Internet integra en las mismas plataformas de distribución a estos sectores, otrora separados." (Becerra y Mastrini, 2015: 66)

"El sistema de medios de comunicación y de industrias convergentes como las telecomunicaciones e Internet ofrecen, en América Latina, una estructura de propiedad fuertemente concentrada con la predominancia de dos grandes conglomerados de telecomunicaciones como Telefónica y Telmex y de grupos multimedios como Globo, Televisa, Cisneros o Clarín.” (Becerra y Mastrini, 2015: 80)

Asimismo, "los principales grupos que operan en América Latina son, en la mayoría de los casos, de origen latinoamericano, con la excepción de capitales españoles en el caso de Telefónica o estadounidenses en el caso de DIRECTV" (Becerra y Mastrini, 2015: 67). Sin embargo, la escala de ingresos de las telefónicas y de DIRECTV es muy superior a la de los multimedios latinoamericanos (Becerra y Mastrini, 2015).

Del mismo modo que a las empresas mediáticas, los gobiernos beneficiaron históricamente a estas corporaciones. De hecho, los niveles de concentración mencionados y el crecimiento exponencial de los principales grupos de medios y telecomunicaciones no podrían haberse logrado sin las facilidades regulatorias (decretos, leyes), políticas (otorgamiento de permisos, licencias, prórrogas) y económicas (subsidios, exenciones impositivas, condonaciones de deudas), que les otorgaron los gobiernos de diverso signo políticos durante décadas (Becerra y Mastrini, 2017).

Además de los gobiernos y las corporaciones, en algunos países de América Latina es también relevante la incidencia de organizaciones para-estatales y criminales como amenazas a libertad de expresión de periodistas y activistas, en medios tradicionales y también en los digitales y redes sociales. En Colombia, por ejemplo, las amenazas a periodistas de organizaciones armadas vinculadas al narcotráfico aumentaron progresivamente desde la firma de los Acuerdos de Paz en 2016 (en 2015 fueron 59; en 2016, 90, en 2017, 129, y en 2018, 200). Los blancos principales son medios comunitarios e indígenas, $y$ van en paralelo con los asesinatos sistemáticos a líderes sociales que denuncian violaciones a derechos humanos en sus regiones, que desde 2016, fueron más de 300 (Fundación para la Libertad de Prensa, 2018). 
María Soledad Segura

Estos problemas para los derechos a la comunicación, se agravan al producirse en sociedades caracterizadas por una profunda desigualdad socioeconómica histórica y estructural, y construidas en base a genocidios y delitos de lesa humanidad, basados en el racismo, el clasismo, y/o diferencias políticas. De hecho, la región no es la más pobre, pero sí la más desigual del mundo (Burchardt, 2012), incluso después de que en los primeros quince años del siglo XXI se registrara una modesta mejora en los niveles de desigualdad y pobreza (Amarante y Colacce, 2018).

Esto se traduce también en desigualdad de acceso y participación en la esfera pública. La esfera pública no es un área igualitaria, sino que reproduce esas desigualdades sociales, culturales, religiosas, económicas, de género, de orientación sexual y políticas de cada país y de la comunidad de naciones.

Se incrementan así los riesgos para la comunicación pública: por un lado, como ya se vio- por el desigual acceso a las tecnologías de conectividad y digitalización y a los datos.

Por otro lado, la inequidad social implica, en última instancia, también profundas discrepancias comunicativas en la esfera pública, potenciadas por las posibilidades de difusión masiva, instantánea y reticular de las redes sociales:

"Las nuevas condiciones de la comunicación pública ponen de manifiesto el colapso de la esperanza moderna de un modelo bien definido y aceptado de decir la verdad basado en la razón. (...) Ahora, en cambio, (...) Las creencias grupales dispersas hacen imposible la aspiración de decir la verdad como un proyecto colectivo guiado por la racionalidad y la facticidad" (Waisbord, 2018: 3).

Con respecto a la vigilancia digital, ni los gobiernos ni las empresas han desarrollado en la región "operaciones masivas y efectivas a gran escala para recopilar, analizar y administrar datos sobre las poblaciones durante el último medio siglo" (Segura y Waisbord, 2019: 6). De este modo, su capacidad para controlar a la población aún no alcanza niveles tan masivos como en los países centrales. 
Vol. XXXIII | N² 2 julio - diciembre 2019 | ISSN 0719-0883 | pp. 198-228

“A pesar de la tradición de controlar a las poblaciones, e incluso si algunos estados de la región han incorporado tecnologías de vigilancia de datos en los últimos años, históricamente no ha habido ambiciosos proyectos estatales de acumulación de datos similares a los que desarrollaron los países centrales en el marco de las políticas de bienestar social, desarrollo militar, geopolítica y operaciones de inteligencia (...) que sentó las bases para la vigilancia de datos contemporánea en los países centrales. Tampoco existe en la región una tradición bien desarrollada de acumulación corporativa de información y cuantificación del consumidor" (Segura y Waisbord, 2019: 6).

Por lo tanto, las principales causas de los problemas para la comunicación y la cultura públicas en la era digital que identifica y enfrenta el activismo por los derechos digitales de América Latina siguen siendo los abusos de poder del mercado y los gobiernos, tal como los fueron históricamente en la región y en el mundo. Además, en algunos países latinoamericanos, se suma con especial relevancia un tercer actor ya mencionado: las organizaciones criminales. Para superar estos problemas, como se muestra a continuación, se organizan de manera autónoma o bien recurren al Estado como aliado para garantizar los derechos vulnerados.

\section{ESTRATEGIAS}

Ante el escenario descrito en apartados previos, ¿qué clases de estrategias desarrolla el activismo por los derechos digitales en América Latina para impulsar sus demandas frente a los poderes gubernamentales, mercantiles y de organizaciones criminales? Estos tipos de estrategias de intervención en lo público, ¿son novedosas o replican antiguos modos del activismo comunicacional y cultural? ¿Son propias de la región o comunes con otros lugares del mundo?

Los activistas digitales y de datos presionan para extender las fronteras de los derechos legalmente reconocidos. Para ello, desarrollan tres tipos de estrategias: 1) de sensibilización pública y pedagógicas para instalar el tema de los derechos digitales como cuestión en la agenda pública y orientar su marco interpretativo desde el paradigma de los derechos humanos; 2) de advocacye incidencia en los gobiernos, organismos internacionales y empresas para que desarrollen regulaciones basadas en derechos humanos; y 3) de desarrollo tecnológico 
María Soledad Segura

alternativo y propio para dar solución a los problemas identificados.

Por un lado, las organizaciones de la sociedad civil que abogan por los derechos digitales, desarrollan estrategias pedagógicas, como la producción de información y la promoción del debate público, para instalar los derechos digitales como un tema en la agenda pública y enmarcar su interpretación en base al paradigma de los derechos humanos; $y$, de manera complementaria, abogan ante gobiernos, organizaciones internacionales y empresas, para que desarrollen regulaciones basadas en los derechos humanos, al redactar propuestas de políticas y proyectos de ley, litigio estratégico o mecanismos administrativos y judiciales para hacer cumplir la aplicación de derechos.

Así, por ejemplo, organizaciones de la sociedad civil como los capítulos regionales de Artículo 19 y las argentinas Fundación Vía Libre y Asociación por los Derechos Civiles abogan por la protección de los derechos civiles en Internet a través de la producción de información, la promoción del debate público, la redacción de propuestas de políticas, la presentación de proyectos de ley al Congreso, el uso de litigios estratégicos y de mecanismos administrativos para hacer cumplir la aplicación de estos derechos.

En materia de derechos culturales en la sociedad digital, un eje de acción es el de las organizaciones por los derechos digitales que impulsan que los Estados desarrollen políticas y dicten regulaciones de libre acceso y software libre.

Hay quienes promueven políticas activas de acceso a la información pública digital, lo que implica que los gobiernos y las empresas dejen estos datos disponibles en línea de modo comprensible y fácilmente utilizable. Además, de seguir insistiendo en las políticas de acceso a la información pública según requerimiento explícito de los/as ciudadanos/as.

Organizaciones como Amnesty International promueven recomendaciones para evitar los ataques y proteger la libertad de expresión en las redes sociales:

“Tanto el Estado como las empresas de redes sociales digitales deberían fortalecer y simplificar las herramientas de denuncia, dar respuesta a denuncias de los usuarios frente a comportamientos abusivos, generar normas de uso claras y transparentes con la participación de las comunidades de usuarios" (Amnesty International-Argentina, 2018: 37). 
Vol. XXXIII | N² 2 julio - diciembre 2019| ISSN 0719-0883 | pp. 198-228

Asimismo, estas organizaciones promueven regulaciones ex ante de neutralidad de la red para evitar discriminación de mensajes y plataformas de internet. Sostienen que es necesario consagrar este principio de manera legal, puesto que las restricciones en el acceso a contenidos representan una amenaza para el derecho a la libertad de expresión de los usuarios (Califano, 2013).

En esa línea, Observacom, Intervozes, Instituto Brasileiro de Defensa do Consumidor, y Desarrollo Digital elaboraron en 2019 el documento “Aportes para una regulación democrática de las grandes plataformas que garantice la libertad de expresión en Internet", en el que ofrecen orientaciones para desarrollar marcos normativos en sintonía con los estándares internacionales de derecho a la comunicación y de los usuarios, y postulan un modelo de coregulación para el establecimiento de criterios de regulación asimétrica y progresiva que tome en cuenta el poder de mercado significativo de las plataformas (Bizberge, 2019).

Finalmente, con respecto a la promoción del acceso igualitario a la conectividad y digitalización, hay organizaciones que impulsan políticas y regulaciones que reconozcan y promuevan, por ejemplo, las redes comunitarias de internet, una gestión social de la conectividad.

Por otro lado, diversos grupos activistas producen un desarrollo tecnológico alternativo y autogestionado para dar una solución autónoma a los problemas identificados, incluso cuando esa solución aún es ilegal. En algunos casos, se trata de colectivos que desarrollan los tres tipos de estrategias y, en otros, de agrupaciones que sólo llevan adelante acciones autónomas.

Así, para combatir la privatización y comercialización de bienes y servicios culturales, y para asegurar su preservación, trabajan en cuatro líneas autogestivas. Una línea consiste en la colaboración para producir conocimiento gratuito como los capítulos en países latinoamericanos de Wikimedia Foundation, que incluye a Wikipedia (la enciclopedia en línea gratuita creada y editada por voluntarios de todo el mundo) y Wikicommons (la biblioteca de fotos gratuita más grande del mundo que contiene ilustraciones, dibujos, videos y música). También desarrollan software libre y abierto, como se ilustra en el ejemplo de los capítulos regionales de Free Software Foundation y el Festival Latinoamericano de Instalación de Software Libre. 
María Soledad Segura

Otro eje de acción es el de poner información públicamente disponible en línea. En ese sentido, digitalizan y publican libros en línea, como el Proyecto Bibliohack Argentino que desarrolla escáneres y materiales de bajo costo con recursos abiertos disponibles en Internet, utiliza sistemas gratuitos de gestión de colecciones y fomenta la creación de redes institucionales de colaboración para preservar el patrimonio cultural. Otros sitios, como el argentino Taringa! Inteligencia colectiva, se basan en publicaciones de sus usuarios (y en la clasificación de popularidad de esas publicaciones) que comparten una amplia gama de tipos de información. Entre toda esa información, también hay enlaces a sitios para la descarga gratuita de material con derechos de autor, resultados de piratería de sitios del gobierno, instrucciones para el pirateo de software y hardware propietario -como el programa Conectar Igualdad, o la tarjeta de transporte público de pasajeros de la Ciudad de Buenos Aires (Red Sube), de Argentina.

Un tercer tipo de acción es la promoción de alternativas a la legislación convencional sobre derechos de autor al ampliar la gama de trabajos creativos disponibles para compartir abiertamente y permitir a los autores decidir los límites de uso y explotación de su trabajo en Internet, como lo ilustran las actividades locales de Creative Commons International, como la ONG Derechos Digitales de Chile. Finalmente, también trabajan en la conservación del trabajo digital y en la conservación de archivos de páginas web y software como patrimonio cultural de la humanidad. No obstante, algunas poblaciones reclaman su derecho a estar desconectadas para preservar sus culturas de los abusos del mercado. Por ejemplo, algunos grupos indígenas se niegan a digitalizar artesanías y telas para evitar el robo corporativo.

En materia de acceso a la información pública y libertad de expresión, algunas organizaciones generan datos originales de manera colaborativa, como el Consorcio Internacional de Periodistas de Investigación que produjo los Papeles de Panamá, en el que participaron muchos periodistas latinoamericanos. También trabajan en producción de información pública el periodismo de investigación y el periodismo de datos que cobraron nuevas dimensiones con las posibilidades tecnológicas. Otro caso es el de las organizaciones de verificación de datos que producen información para monitorear el discurso público, como Chequeado en Argentina, UYCheck en Uruguay o El Observador en Honduras, y varios proyectos de verificación de hechos lo hicieron durante las recientes campañas electorales en Costa Rica, 
PERSONA\&SOCIEDAD|VOL.XXXIII||N²|217

Vol. XXXIII | N² 2 julio - diciembre 2019 | ISSN 0719-0883 | pp. 198-228

Colombia, Brasil y México.

Finalmente, hay grupos que hackean información pública ocultada por gobiernos o corporaciones y la ponen disponible en línea. Por otra parte, frente a la multiplicación de identidades falsas que se usan para difundir información falsa o engañosa, y para hostigar a usuarios por sus expresiones públicas, académicos hacen análisis estadístico para identificar trolls (como los horarios en que trabajan).

Varias organizaciones de la sociedad civil han trabajado para superar las desigualdades digitales, como las redes comunitarias de Internet como Altermundi y Atalaya Sur en Argentina, Coolab en Brasil, Rhizomática y Telecomunicaciones Indígenas Comunitarias en México, que usan software libre y hardware abierto para que pueda ser apropiado por las comunidades; y proyectos de reciclaje de deshechos electrónicos como Computadores para Educar en Colombia que generan empleos, contribuyen a una gestión sostenible de los desechos electrónicos y redistribuyen los equipos.

Por lo tanto, se verifica que el activismo por los derechos digitales en América Latina implementa tácticas y estrategias que no son completamente diferentes de las utilizadas por movimientos similares en el Norte. Por el contrario, suelen ser similares a nivel global, salvo la atención a la brecha digital que es específicamente regional o de los países periféricos (Segura y Waisbord, 2019).

Por otra parte, el tipo de acciones desarrolladas por el activismo latinoamericano por los derechos digitales tampoco son diferentes de las implementadas por las organizaciones que impulsaban reformas del sistema de medios tradicionales (Segura y Waisbord, 2016) o que desarrollaron sus propios medios (Segura, 2018). Abogan para que los Estados garanticen nuevos derechos de comunicación relacionados con la digitalización de la información y reformen las políticas de conectividad y de producción y manejo de datos, y para incidir en la opinión pública de la misma manera que los activistas mediáticos lo hicieron con las políticas de comunicación audiovisual. Mientras tanto, también desarrollan de manera autogestiva -e incluso no legalmente reconocida- iniciativas que permiten el acceso y ejercicio de los derechos que reclaman hasta tanto sean reconocidos por los estados, tal como lo habían hecho las estaciones de radios y televisoras comunitarias desde mediados del siglo XX hasta que comenzaron a ser reconocidas legalmente en algunos países en la 
María Soledad Segura

primera década del siglo XXI.

\section{ORGANIZACIONES}

El marco interpretativo, las demandas presentadas, los problemas identificados y las estrategias desarrolladas fueron llevadas adelante por sujetos colectivos. ¿Qué características tienen las organizaciones de activistas por los derechos digitales en Latinoamérica? ¿En qué medida son similares a o se distinguen de las de los países centrales?

Estas organizaciones están interesadas en promover políticas públicas para regular las prácticas corporativas de extracción de datos y frenar el poder de los estados en asuntos relacionados con el acceso, producción, protección y administración de datos, y el acceso a la tecnología a través de software libre y hardware abierto.

Estas nuevas organizaciones de derechos digitales surgieron en la década de 2000 en la región para abogar por políticas de Internet que protegen los derechos civiles: especialmente los derechos relacionados con la privacidad, la seguridad, la neutralidad de la red, la libertad de expresión y el acceso a la información. Una de las primeras organizaciones latinoamericanas de este tipo es la Fundación Argentina Vía Libre, que se creó en 2000, 15 años después de uno de los primeros movimientos de datos similares en países centrales como Free Software Foundation. Esta brecha temporal es comprensible porque el desarrollo tecnológico y la penetración de la digitalización y la conectividad masivas sucedieron más tarde en América Latina.

Dentro de estas organizaciones, es compleja la relación entre los/as expertos/as técnicos/as y los/as demás activistas, como ya reseñan algunos trabajos (por ejemplo: Chenou y Cespeda-Másmela, 2019). Hay una brecha grande de conocimiento y competencias tecnológicas entre unos/as y otros/as. Además, es un campo en el que es conocida la figura del activista que actúa como "llanero solitario", debido al altísimo nivel de conocimiento requerido y porque el tipo de herramienta lo permite. Esto es más frecuente en los países centrales (como los casos de Swartz, Snowden, o Manin), pero también se registran casos de activistas hackers en América Latina, por ejemplo, para mostrar los bugs de las máquinas de voto electrónico que violan el secreto del voto. Estos activistas individuales suelen exponerse a mayores riesgos de 
PERSONA\&SOCIEDAD|VOL.XXXIII||N²|219

Vol. XXXIII | Nº 2 julio - diciembre 2019| ISSN 0719-0883 | pp. 198-228

represión.

En los últimos años estas organizaciones se han articulado de diversas maneras para unir recursos, capacidades, conocimientos, herramientas, relaciones y, por lo tanto, también poder de incidencia. Conforman redes con diversos fines a corto, mediano o largo plazo. Así, por ejemplo, Coding Rights de Brasil, Hiperderecho de Perú, Fundación Karisma de Colombia, InternetLab de Brasil, IPANDETEC de Panamá, Red en Defensa de los Derechos Digitales (R3D) de México y TEDIC de Paraguay integran una red que trabaja en conjunto y realiza publicaciones como el "Reporte de la situación de América Latina sobre la Violencia de Género ejercida por Medios Electrónicos”. Otras organizaciones de activistas establecen alianzas entre ellas y con medios de comunicación de masas y agencias de noticias, como lo hicieron las organizaciones de fact-checking en diversos países de la región ante las elecciones nacionales: Comprova en Brasil, ReversoAr en Argentina, Verificado en Uruguay y Chilecheck en Chile, entre otras.

Estos grupos mantienen diversas relaciones con el Estado y el mercado. Así como hay organizaciones autonomistas que se mantienen más alejadas de estos otros dos sectores poderosos, otras mantienen vínculos complejos. Las organizaciones de verificación del discurso público, por ejemplo, se aliaron con empresas de medios y agencias de noticias comerciales. Otro caso muy particular es Taringa!: una red social que capta publicidad (como lo hacen los grandes jugadores del mercado digital), pero es nacional, alternativa $y$ contracultural. Es una corporación pequeña (en términos relativos) y no está asociada a ninguna otra, razón por la cual es menos permeable a la presión de quienes tienen los copyright de los contenidos. Además, como no introduce filtros de ningún tipo ni censura ninguna información, se constituyó como una plataforma en la que se concentra cierto tipo de contenidos vinculados a la ampliación de derechos digitales: se comparten, por ejemplo, links a sitios piratas, se indica cómo hackear software y hardware, etc. Más aún: con el fallo judicial a favor que consiguieron (al que se hace referencia en la sección siguiente), nadie podrá ya hacerles bajar esos contenidos compartidos.

Muchas de las organizaciones latinoamericanas de activistas digitales son capítulos nacionales de organizaciones internacionales como Wikimedia o Creative Commons. Otras son organizaciones no gubernamentales internacionales o regionales de derechos humanos que empezaron a incluir los 
María Soledad Segura

derechos digitales en sus agendas, como la Asociación por los Derechos Civiles o el Centro de Estudios Legales en Argentina, Artículo 19 Internacional en Brasil o México, o Amnistía Internacional en muchos países de la región. Algunas otras son organizaciones locales, pero en su mayoría integran alianzas internacionales, como el costarricense Código Sur que también tiene parte de su equipo en otros países latinoamericanos, Fundación Vía Libre, o el sitio argentino Taringa! Inteligencia Colectiva. Por lo tanto, los orígenes internacionales o las alianzas internacionales de las organizaciones latinoamericanas de activismo digital y de datos son muy relevantes. Se podría razonablemente argumentar que esto se debe a la expansión transnacional y los efectos de la tecnología, su origen occidental y su desarrollo temprano en países con economías fuertes. En consecuencia, no es posible afirmar la existencia de una específica práctica latinoamericana de resistencia y de incidencia vinculada a los derechos digitales, diferente a la de los países centrales, sino que se vincula con un movimiento global.

El activismo por los derechos digitales se produce simultáneamente con la movilización de las organizaciones que impulsaron reformas en las políticas de comunicación (Segura y Waisbord, 2016; Segura, 2018) y cultura (Prato y Segura, 2018), que lograron inéditos niveles de incidencia. Al compartir, en términos generales, el marco normativo de los derechos humanos, su incidencia se potencia mutuamente $y$, en no pocas ocasiones, encuentran sinergias para estrategias conjuntas como, por ejemplo, la organización mexicana Rizhomática que desarrolla telefonía celular comunitaria y redes comunitarias de internet, basada en el modelo de las radios comunitarias.

Además, se inicia en el momento de auge de gobiernos progresistas o "a la izquierda del centro" (Panizza, 2005: 716), durante los primeros quince años del siglo XXI en muchos de los países de la región. Como se muestra en la siguiente sección, aunque no fueron las únicas, estas fuerzas políticas fueron ideológicamente más proclives a avanzar en políticas de acceso abierto, software libre y neutralidad de la red (Freedman, 2008).

En síntesis, como la red es global, los problemas y el modo de resolverlos también. Por eso, muchas organizaciones por los derechos digitales en la región son capítulos nacionales de organizaciones de la sociedad civil internacional, o son parte de alianzas internacionales que surgieron en las últimas dos décadas (Segura y Waisbord, 2019). Sin embargo, también hay diferencias temporales y 
Vol. XXXIII | N² 2 julio - diciembre 2019 | ISSN 0719-0883 | pp. 198-228

de magnitud en los problemas, como el retraso en el acceso a las tecnologías de la digitalización y la conectividad. Por ende, el activismo por los derechos digitales en América Latina aparece 20 años después que en los países centrales y hay muchas organizaciones que trabajan la cuestión del acceso digital que sigue siendo un problema central.

Este activismo que promueve los derechos digitales y de datos es equivalente a los movimientos que abogaron por los derechos de comunicación en relación con las políticas de televisión y radio que tuvieron una participación e impacto inusuales en América Latina durante las primeras décadas del siglo XIX (Segura \& Waisbord, 2016). En la era analógica, también había activistas que impulsaban el acceso a la información pública y la libertad de expresión, pero tenían diferentes herramientas y desafíos. La novedad está dada por la masificación, la instantaneidad y las posibilidades de propagación horizontal en toda la red, gracias a un crecimiento exponencial en la potencia de cómputo, y la posibilidad de almacenamiento digital permanente.

\section{INCIDENCIA}

¿Qué impacto ha tenido el activismo latinoamericano por los derechos digitales en la formulación de políticas estatales basadas en ellos (leyes y regulaciones, fallos judiciales y programas del Poder Ejecutivo) y en promover estos derechos de manera autogestiva?

Por un lado, las organizaciones activistas del continente incidieron en los Estados, especialmente en su Poder Legislativo para sancionar nuevas leyes referidas a derechos civiles y neutralidad de la red, y acceso a bienes culturales digitales. Estas organizaciones cívicas participaron en el proceso que resultó en la aprobación reciente en varios países de legislación para proteger los derechos civiles y garantizar la neutralidad de la red, como el Marco Civil da Internet de 2014 en Brasil. En Perú, en 2016, y luego de más de un año de consultas, el Organismo Supervisor de Inversión Privada en Telecomunicaciones (OSPITEL) aprobó el Reglamento de Neutralidad de Red, que impide "la gestión arbitraria del tráfico, el filtro y/o bloqueo arbitrario de servicios y aplicaciones y la diferenciación arbitraria en la oferta de comercial de productos de acceso a Internet" (Observacom, 2017: 1), a través de la restricción de acceso, priorización, limitación de calidad o funcionalidad y cobro adicional (artículo 
María Soledad Segura

32). Chile también cuenta con un marco específico sobre neutralidad de red: la Ley 20453 desde 2010. En cambio, en Argentina y México, el principio de neutralidad está consagrado de modo general dentro de las leyes de telecomunicaciones sancionadas en 2014 en ambos casos: la Ley Argentina Digital y la Ley de Telecomunicaciones en México (Observacom, 2017).

Otro logro de incidencia en las normativas, ha sido el de las organizaciones argentinas Centro de Estudios Legales y Sociales, Asociación de los Derechos Civiles, Asociación Civil por la Igualdad y la Justicia, Fundación Vía Libre y otras que, en 2018, consiguieron que los/as legisladores/as retiren del proyecto de reforma del Código de Procesamiento Penal de Buenos Aires y del Código Federal de Procesamiento Penal la autorización del uso de piratería gubernamental para permitir "medidas especiales de investigación", "como el uso de malware por parte del gobierno en investigaciones criminales" (Electronic Frontier Foundation, 2018: 1).

Además, la Ley de Repositorios de Acceso Abierto Institucional Digital de 2013 en Argentina garantiza el acceso público al conocimiento académico, y la Ley de Software Libre y Formato Abierto sancionada en Uruguay en 2014 promueve el uso de software libre en el país.

Por otra parte, también han contribuido a políticas de datos abiertos desarrolladas por los Poderes Ejecutivos, como la Estrategia Digital Nacional de México implementada entre 2014 y 2018, o la decisión de adoptar software abierto de la administración ecuatoriana en 2009. También contribuyeron con la soberanía tecnológica y seguridad nacional en el proyecto de desarrollar su propio correo electrónico cifrado en Brasil para evitar el espionaje digital en 2014.

Además, gracias a la tarea pedagógica y de advocacy del activismo por los derechos digitales, algunas decisiones judiciales comienzan a reconocer la prioridad del derecho a comunicarse por Internet sobre otros derechos. Por ejemplo, la Justicia argentina declaró inocentes a los fundadores de Taringa! acusados de violación de propiedad intelectual. Consideraron que si ellos hubieran verificado si sus usuarios tenían los derechos de propiedad intelectual para compartir una publicación y, eventualmente, desaceleraban la publicación, habrían ejercido censura previa. 
La sociedad civil también contribuyó para que la Justicia proteja los derechos a la privacidad y la no vigilancia por gobiernos ni empresas. El Tribunal de Justicia de São Paulo ordenó que Via Quatro, empresa concesionaria del metro de esa ciudad, suspendiera la recopilación de datos de pasajeros del metro, utilizando anuncios en trenes que rastreaban expresiones faciales y rasgos del usuario, a pedido del Instituto Brasileño de Protección al Consumidor y la Red Latinoamericana de Vigilancia, Estudios de Tecnología y Sociedad, en defensa de los derechos a la privacidad de alrededor de 600.000 brasileños que utilizan el sistema de transporte público todos los días. En Chile, el grupo latinoamericano Derechos Digitales incidió para que el fiscal de la Unidad de Alta Complejidad de La Araucanía confirmara el enjuiciamiento de funcionarios de Inteligencia por obstrucción de la justicia mediante la presentación de pruebas falsas basadas en chats electrónicos para incriminar a miembros de la comunidad mapuche como integrantes de una organización terrorista, en 2017 (Electronic Frontier Foundation, 2018).

Por otro lado, las organizaciones latinoamericanas también han conseguido que las corporaciones de redes sociales impulsen autorregulaciones muy controvertidas por sus efectos nocivos para la libertad de expresión. Esto con el fin de controlar la distribución de desinformación, noticias falsas, hechos alternativos, y la propagación de identidades falsas. A partir de las críticas recibidas en este sentido, empresas de redes sociales tomaron medidas para eliminar fake accounts (Twitter), censurar contenidos considerados ofensivos (Facebook) o restringir las posibilidades de distribución de información falsa (Whatsapp). Brasil, por ejemplo, es uno de los diecisiete países donde Facebook cuenta con verificadores externos de datosque intentan eliminar la desinformación publicada en su sección de noticias. Google y esa red social también han colaborado en una iniciativa llamada Comprova, que reúne a veinticuatro redacciones brasileñas con el objetivo de desmentir enlaces, videos e imágenes (Tardáguila, Benevenuto y Ortellado, 2018).

Asimismo, son considerables los logros de las organizaciones ciudadanas en términos de expansión del uso de licencias de Creative Commons, la consolidación y crecimiento de Wikipedia y del software libre. Esto ha sido ayudado por el interés de Estados y de empresas en la incorporación de esta tecnología gratuita y efectiva. 
María Soledad Segura

Finalmente, es de destacar que la actividad pedagógica de las organizaciones por los derechos digitales ha contribuido decisivamente en la creciente conciencia social sobre la relevancia de la seguridad informática para la protección de datos personales, y sobre la necesidad de verificación de contenidos e identidades en la deliberación pública. No obstante, es menor el conocimiento sobre los modos para lograrlo. Aparentemente, es menos relevante la conciencia de la necesidad del libre acceso a bienes y servicios culturales y al software libre y hardware abierto y no propietario; así como a la información pública. No obstante, esto requeriría mayor estudio para poder ser comprobado.

Se verifica entonces que el activismo por los derechos digitales en la región está contribuyendo a ampliar el reconocimiento estatal, empresarial y social de los derechos digitales.

\section{CONSIDERACIONES FINALES}

En síntesis, frente a los problemas en el uso de las tecnologías de la digitalización y la conectividad en el capitalismo digital, las organizaciones de activistas por los derechos digitales en América Latina son actores progresistas de la sociedad civil, que tienen por propósito garantizar los derechos humanos a la comunicación (acceso a la información y libertad de expresión), la cultura y la privacidad; que entienden que, para ello, es necesario reducir los abusos de poder de gobiernos y empresas en la comunicación pública digital; y que, con ese fin, desarrollan dos tipos de estrategias: por un lado, presionan para la formulación de políticas públicas basadas en derechos y, por otro, desarrollan prácticas autogestivas para ejercer estos derechos de hecho, estén o no reconocidos legalmente.

El análisis realizado sobre problemas que enfrenta, las demandas, el marco interpretativo, las estrategias, las características de las organizaciones y el impacto del activismo por los derechos digitales en América Latina, permite afirmar lo siguiente:

a. Enfrenta problemas antiguos para la comunicación pública, la cultura y la privacidad, aunque esos problemas adquieren nuevas dimensiones debido a las nuevas posibilidades tecnológicas. Los antiguos problemas, implican identificar también viejos y conocidos oponentes poderosos: el 
Vol. XXXIII | N² 2 julio - diciembre 2019| ISSN 0719-0883 | pp. 198-228

Estado y el mercado, cuyos abusos distorsionan la comunicación y la cultura pública y la privacidad personal. Como la red es global, los problemas también son globales. No obstante, la red global presenta también problemas locales, especialmente, la desigualdad en el acceso, y el retraso en el acceso, debido al desinterés del capital por poblaciones periféricas. Por estas diferencias de acceso, también hay diferencias temporales y de magnitud en los problemas. Finalmente, los problemas globales también adquieren, en algunos casos, particularidades regionales debido a la diferencia de contextos e historias locales, como la extensión y masividad de la vigilancia digital y de datos, así como las diferenciadas condiciones para las campañas de desinformación.

b. Como la mayoría de los problemas no son nuevos, el activismo digital latinoamericano recupera también antiguos marcos normativos referidos a los derechos humanos a la comunicación, la cultura y la privacidad, pero establece nuevas precisiones para enfrentar las particularidades de la era digital. Esos marcos normativos son occidentales, originados en Europa y Estados Unidos. Sin embargo, algunas de las innovadoras precisiones realizadas tienen origen en organizaciones de la región que las formulan teniendo en cuenta los contextos e historia locales.

c. Los antiguos problemas, requieren también conocidas estrategias: incidir en políticas públicas (leyes y regulaciones, sentencias judiciales, medidas administrativas, etc.), luchar por el reconocimiento $y$ ampliación de derechos, por un lado, junto a la realización de prácticas autogestivas, ejerciendo el derecho de facto (incluso cuando esas prácticas sean actualmente ilegales).

d. Dado que la red y sus problemas son globales, la mayoría de las organizaciones por los derechos digitales en la región son capítulos regionales de organizaciones internacionales o integran redes internacionales. Sin embargo, dadas las desigualdades de acceso en la región, el activismo por los derechos digitales en América Latina aparece 20 años después que en los países centrales. Además, hay muchas organizaciones que trabajan la cuestión del acceso digital. 
María Soledad Segura

Por lo tanto, como ya hemos señalado (Segura y Waisbord, 2019), el activismo por los derechos digitales en América Latina tiene en cuenta los problemas y las condiciones locales y, al mismo tiempo, está abierto a posicionamientos y estrategias desarrollados en otras regiones del mundo. Al igual que otros movimientos por los derechos de comunicación en América Latina, las formas actuales de ciudadanía digital abarcan diferentes tradiciones políticas y legales al enfrentar los aspectos locales y globales del capitalismo digital. El activismo latinoamericano por los derechos digitales y de datos recorre los caminos ya trazados por el activismo comunicacional y cultural en la era analógica en sus modalidades de intervención en lo público, y su conflictiva relación con el poder estatal y el mercantil. Por lo que la disputa por lo digital no modifica -en lo esencial- las dinámicas y los poderes estructurales de la comunicación pública, sino que los actualiza.

\section{BIBLIOGRAFÍA}

Amarante, V. \& Colacce, M. (2018). ¿Más o menos desiguales? Una revisión sobre la desigualdad de los ingresos a nivel global, regional y nacional, Revista CEPAL, 124: 7-34.

Amnesty International-Argentina (2018). 'El debate público limitado: trolling y agresiones a la libertad de expresión de periodistas y defensores de DDHH en Twitter Argentina'. Recuperado de: $\quad$ https://amnistia.org.ar/wp-content/uploads/delightfuldownloads/2018/03/online-pre1.pdf

Amnesty International-United Kingdom (2017). 'Black and Asian women MPs abused more online'. Recuperado de: https://www.amnesty.org.uk/online-violence-women-mps

Becerra, M. \& Mastrini, G. (2015). Concentración y convergencia de medios en América Latina, Ensambles, 2(3): 64-83.

Bizberge, A. (2019). Cómo regular a las grandes plataformas y proteger a los usuarios. Una propuesta de la sociedad civil latinoamericana, Montevideo: Observacom.

Burchardt, H. J. (2012). Por qué América Latina es tan desigual. Tentativa de explicación desde una perspectiva inusual, Nueva Sociedad, 239: 137-150.

Califano, B. (2013). Políticas de Internet: la neutralidad de la red y los desafíos para su regulación, Revista Eptic, 15 (3): 19-37.

CEPAL (2016). Estado de la banda ancha en América Latina y el Caribe, Santiago de Chile: United Nations/CEPAL y German Cooperation Deustche Zusammenarbeit.

Chenou, J. M. \& Cespeda-Másmela, C. (2019). ‘\#NiUnaMenos: Data activism from the Global South', Television \& new media, 20(4): 396-411. 
Vol. XXXIII | N² 2 julio - diciembre 2019 | ISSN 0719-0883 | pp. 198-228

Coalizão Direitos na Rede (2017). Open letter from the Latin America and Caribbean civil society representatives on the concerns around the discourse about fake news and elections, Genova: Foro de Gobernanza de Internet.

Electronic Frontier Foundation (2018). 'Where governments hack their own people and people fight back'. Recuperado de: https://www.eff.org/es/deeplinks/2018/12/wheregovernment-hack-their-own-people-and-people-fight-back-latin-american

FLIP (2018). Prensa acorralada: un juego de violentos y poderosos, Bogotá: FLIP.

Fraser, N. (2006). Reinventar la justicia en un mundo globalizado, New Left Review, 36: 31-50.

Freedman, D. (2006). Dynamics of power in contemporary media policy-making, Media, Culture \& Society, 28(6): 907-923.

(2008). The politics of media policy, Cambridge: Polity Press.

Gutiérrez, M. (2018). Data activism and social change, Londres: Palgrave/Pivot.

Lesaca, J. (2019). 'Hay que desconfiar de lo que llegue por Whatsapp', Diario Correo, Lima.

Mariscal, J. \& Rivera, E. (2007). 'Regulación y competencia en las telecomunicaciones mexicanas', Serie Estudios y Perspectivas, 83, México: Naciones Unidas/CEPAL.

Mastrini, G. \& Aguerre, C. (2009). 'La regulación de las telecomunicaciones y el desarrollo de la banda ancha. Implicancias para la región andina', Asociación para el Progreso de las Comunicaciones.

Recuperado

de:

https://www.apc.org/sites/default/files/CILACInvestigacionesIntroduccion 20090722 0.pdf

Milan, S. \& van der Velden, L. (2016). The Alternative Epistemologies of Data Activism, Digital Culture and Society, 2(2): 57-74.

Millaleo, S. \& Velasco, P. (2013). Activismo digital en Chile. Repertorios de contención e iniciativas ciudadanas, Santiago de Chile: Fundación Democracia y Desarrollo.

Observacom (2017). Perú aprueba reglamento de neutralidad de red, Montevideo: Observacom.

Oszlak, O. \& O'Donnell, G. (1984). 'Estado y políticas estatales en América Latina’. En Flores, G. \& Nef, J. (eds.) Administración pública:Perspectivas críticas, Buenos Aires: ICAP.

Panizza, E. (2005). Unarmed utopia revisited: the resurgence of left-of-centre politics in Latin America, Political Studies, 53: 716-734.

Prato, A. V. \& Segura, M. S. (2018). Estado sociedad civil y políticas culturales. Rupturas y continuidades entre 2003 y 2017 en Argentina, Buenos Aires: RGC Ediciones.

REGULATEL (2016). 'Estudio comparado. Modelos de regulación en el sector de las telecomunicaciones y su relación con la defensa de la competencia en los países miembros de REGULATEL'. Recuperado de: http://regulatel.org/w/wpcontent/uploads/2018/11/Estudio-comparado-Modelos-de-regulaci\%C3\%B3n-en-elsector-de-las-telecomunicaciones-y-su-relaci\%C3\%B3n-con-la-defensa-de-lacompetencia-en-los-pa\%C3\%ADses-miembros-de-Regulatel-2016..pdf 
María Soledad Segura

Rivera, E. (2004). Regulación y competencia de las telecomunicaciones en Centroamérica: un estudio comparativo, Serie Estudios y Perspectivas, 22, México: Naciones Unidad/CEPAL.

Segura, M. S. (2018). De la resistencia a la incidencia. Sociedad civil y derecho a la comunicación en Argentina, Buenos Aires: Ediciones UNGS.

Segura, M. S. \& Waisbord, S. (2016). Media movements. Civil society and media policy reform in Latin America, Londres: Zed Books.

Segura, M. S. \& Waisbord, S. (2019). 'Between data capitalism and data citizenship', Television \& new media, 20(4): 1-8.

SITEAL (2012). La brecha digital en América Latina, París: OEI/OEA/UNESCO.

Sorj, B. (2010). Usos, abusos y desafíos de la sociedad civil en América Latina, Buenos Aires: Siglo XXI.

Tardáguila, C; Benevenuto, F. \& Ortellado, P. (2018). 'WhatsApp para contener las noticias falsas en las elecciones brasileñas', New York Times en Español, 18 de octubre.

Tarrow, S. (1977). Poder en movimiento. Movimientos sociales, acción colectiva y política de masas en el estado moderno, Madrid: Alianza.

Waisbord, S. (2018). The elective affinity between post-truth communication and populist politics, Communication Research and Practice, 4(1): 17-34.

$\mathrm{Wu}, \mathrm{T}$. (2003). Network neutrality, broadband discrimination, Journal of Telecommunications and High Technology Law, 1(2): 141-179. 